\title{
Monitoring ocular discomfort using a wrist-mounted electronic logger
}

DOI:

10.1016/j.clae.2020.02.010

\section{Document Version}

Accepted author manuscript

Link to publication record in Manchester Research Explorer

\section{Citation for published version (APA):}

Read, M. L., Navascues-cornago, M., Keir, N., Maldonado-codina, C., \& Morgan, P. B. (2020). Monitoring ocular discomfort using a wrist-mounted electronic logger. Contact Lens and Anterior Eye.

https://doi.org/10.1016/j.clae.2020.02.010

\section{Published in:}

Contact Lens and Anterior Eye

\section{Citing this paper}

Please note that where the full-text provided on Manchester Research Explorer is the Author Accepted Manuscript or Proof version this may differ from the final Published version. If citing, it is advised that you check and use the publisher's definitive version.

\section{General rights}

Copyright and moral rights for the publications made accessible in the Research Explorer are retained by the authors and/or other copyright owners and it is a condition of accessing publications that users recognise and abide by the legal requirements associated with these rights.

\section{Takedown policy}

If you believe that this document breaches copyright please refer to the University of Manchester's Takedown Procedures [http://man.ac.uk/04Y6Bo] or contact uml.scholarlycommunications@manchester.ac.uk providing relevant details, so we can investigate your claim.

\section{OPEN ACCESS}




\title{
Monitoring ocular discomfort using a wrist-mounted electronic logger
}

\author{
Michael L. Read ${ }^{\mathrm{a}, *}$, Maria Navascues-Cornago ${ }^{\mathrm{a}}$, Nancy Keir ${ }^{\mathrm{b}}$, Carole Maldonado-Codina ${ }^{\mathrm{a}}$, Philip B. \\ Morgan $^{\mathrm{a}}$ \\ ${ }^{a}$ Eurolens Research, Division of Pharmacy and Optometry, Faculty of Biology, Medicine and Health, The University of \\ Manchester, Manchester, M13 9PL \\ ${ }^{b}$ CooperVision Inc., 6150 Stoneridge Mall Rd, Pleasanton, Calafornia, USA 94588
}

\begin{abstract}
Purpose: To investigate ocular discomfort during contact lens wear using a wrist-mounted electronic 'lens awareness logger' (LAL). Methods: Thirty symptomatic contact lens wearers wore study contact lenses for three days. On the first two days, two lens types which are known to differ in end-of-day comfort (lens A: senofilcon A and lens B: balafilcon A), were worn as a matching pair (randomised order). On day three, a pair of lens B were worn. On each day, the participant wore a LAL. On day one and two, the participant pressed a button on the LAL whenever they became aware of their lenses due to discomfort. On day three, the participant used a multiple click protocol ( $1=$ mild awareness to $3=$ severe awareness). Results: LAL events were similar on days one and two (17.3 vs. 15.8 events per day). There were significantly more LAL events for lens B (21.6 events per day) in comparison with lens A (11.6 events per day) $(\mathrm{p}=0.006)$. The LAL event profile highlighted peaks in awareness following lens application and towards the end of the wearing cycle. Comparison of the LAL event profile for the two lens types showed significant differences in lens awareness, particularly in the first half of the wearing cycle. LAL events on day 3, showed a uniform distribution of single and double clicks through the day, but a marked peak in triple clicks in the last two hours of lens wear. Conclusion: The LAL was able to differentiate between the study lenses and demonstrated differences in their LAL event profiles. Lens awareness associated with discomfort appeared to increase not only in frequency, but also in intensity towards the end of the wearing cycle. The ability of the LAL to track lens awareness suggests it is likely to be a useful tool in furthering understanding of ocular discomfort.
\end{abstract}

Keywords: Contact lens, Comfort, Discomfort, Lens Awareness Logger, End of day, Wearable.

\section{Introduction}

The ocular surface is the most highly innervated tissue of the human body, with the density of pain 3 receptors in the cornea around 300-600 times greater than the skin [1]. Given this, even mild disturbances

\footnotetext{
* Corresponding author.

Email address: michael.read-3@manchester.ac.uk (Michael L. Read)

${ }^{1}$ Conflict of interests: None.

${ }^{2}$ Funding statement: This study was funded by CooperVision Inc.

${ }^{3}$ Acknowledgements: The authors acknowledge the assistance of clinical, logistical and administrative colleagues at Eurolens Research in the acquisition of data for this study. 
to the ocular surface due to surgery, infection, mechanical or chemical irritation can result in the sensation of pain [2]. Eye care practitioners and researchers typically use questionnaires and/or visual analogue scales to assess ocular surface pain [3,4]. Typically these metrics are assessed in a clinical setting and rely on the patient's recollection of symptoms, with the potential for recall bias $[5,6]$. To more accurately characterise changes in ocular comfort with time and minimise the effect of recall bias, a range of approaches have been developed where such grading scores and/or questionnaires are captured outside of the clinic environment. This typically involves prompting the patient to complete a grading scale and/or questionnaire for ocular comfort at key time-points, typically with the use of a paper diary [7], via text messaging [8] or via the internet [9]. Although this allows the capture of ocular comfort data in the real world, there is often still a requirement for recollection of symptoms, the quality of the data is not always assured (e.g. poor compliance particularly with paper diaries [10]) and the patient is required to convert their issues with ocular comfort in to an arbitrary numeric scale. This can be particularly challenging in the fields of dry eye disease and contact lens wear, where symptoms are known to vary significantly through the day depending on a range of factors including environment, application of ocular lubricants, blink rate and visual task. For contact lens wearers and patients with mild dry eye disease, it is common for these episodes of discomfort to be transient, with prolonged periods with no symptoms. In order to address the limitations of existing approaches, the use of wearable technology offers a number of potential advantages including the ability to capture data in real time. Wearable technology in medicine is a rapidly growing area of research and is becoming an integral part of personal analytics, assessment of physical status, reading physiological parameters and informing a schedule for medication $[11,12]$. Previous work has demonstrated that a novel wearable device for subjective reporting of contact lens discomfort - the Lens Awareness Logger (LAL) - was able to provide useful information on the clinical performance of contact lenses [13]. With this type of system, the perfect contact lens product or dry eye treatment would result in no episodes of ocular discomfort being logged, with increasing episodes indicative of a less optimal solution. This original LAL device allowed accurate logging of awareness events through the lens wearing cycle [13], but suffered from a number of limitations including that (i) it was attached to clothing rather than worn and thus could become separated from the participant, (ii) there was no way of checking compliance with the use of device, (iii) the device was not waterproof or robust to adverse environments, and (iv) the device was not always readily available to the participant (i.e. they had to remember where they had attached it to their clothing). It was therefore decided to move towards a wrist-mounted LAL device, which could log episode of lens-associated discomfort (termed lens awareness events) via a front-facing button, whilst also monitoring the other internal sensors (temperature, light and motion sensors) to confirm if the participant was compliant with instructed usage. The work described in this paper investigated if the wrist-mounted LAL device could detect differences in subjective lens awareness in a group of symptomatic contact lens wearers, when two brands of contact lenses, which have previously been reported to differ in end of day comfort (Lens A: senofilcon A and Lens B: balafilcon 
A $[14,15])$ were worn. Furthermore, to ascertain if participants could provide more detailed information about the intensity of any lens-associated discomfort, participants used a more complex reporting system (multi-click protocol) on the final study day.

\section{Methods}

\subsection{LAL device and clinical use}

The wrist-mounted electronic device used in this study was a GeneActiv logger (Activinsights Ltd, Kimbolton, UK), which is typically used as an activity monitor and has been used in a wide range of clinical research studies $[16,17,18,19]$. This event logger was chosen as it is wrist mounted, includes movement/temperature/light sensors and has a event logging button with LED light confirmation (Figure 1). The wrist-mounted nature of the device allowed it to be worn by the study participants in a minimally invasive manner, ensuring it was immediately available to the participants at all times. The environmental sensors (light, temperature and movement) allowed confirmation as to whether the device had been worn continuously during contact lens wear. The event logging button is located on the front surface of the device, allowing the convenient activation of the button during wear, resulting in a time/date logged recording for each button-press event. The LAL device was configured to sample all of the internal sensors at $10 \mathrm{~Hz}$, allowing it to be issued to the study participant for up to two-weeks, without requiring data download or recharging of the internal battery. Prior to clinical testing, the LAL devices were tested to ensure they were correctly logging button presses. This involved each LAL device being worn for a six hour period, with the button pressed when prompted by a hourly alarm. All LAL devices correctly logged the hourly button presses, with no unintended button presses recorded.

A LAL device was issued to each of the study participants, with the participants instructed to put on the LAL device immediately prior to lens application and remove the LAL device immediately following lens removal. To ensure compliant use of the LAL device, the participant received one-to-one training with the investigator and were issued with written instructions. During wear, the participant was instructed to follow a single-click protocol on study day 1 and 2, and a multiple-click protocol on study day 3. For the single-click protocol, the participant was instructed to click the button on the LAL device whenever they became aware of the contact lens due to reduced comfort (in this study termed 'lens awareness'). On day 1 and 2 , the participants were informed that if they pressed the button more than once in a one-minute period then these later presses would be ignored during analysis (to provide sufficient time for the participant to forget about the reduction in contact lens comfort). On day 3 , the participants were asked to follow a similar process, but for each episode of lens awareness they were instructed to grade its severity using a multiple click protocol (1 click $=$ mild awareness, 2 clicks $=$ moderate awareness, 3 clicks $=$ severe awareness $)$. The definition of 'mild', 'moderate' and 'severe' awareness was a subjective opinion of each individual participant. Multiple button 


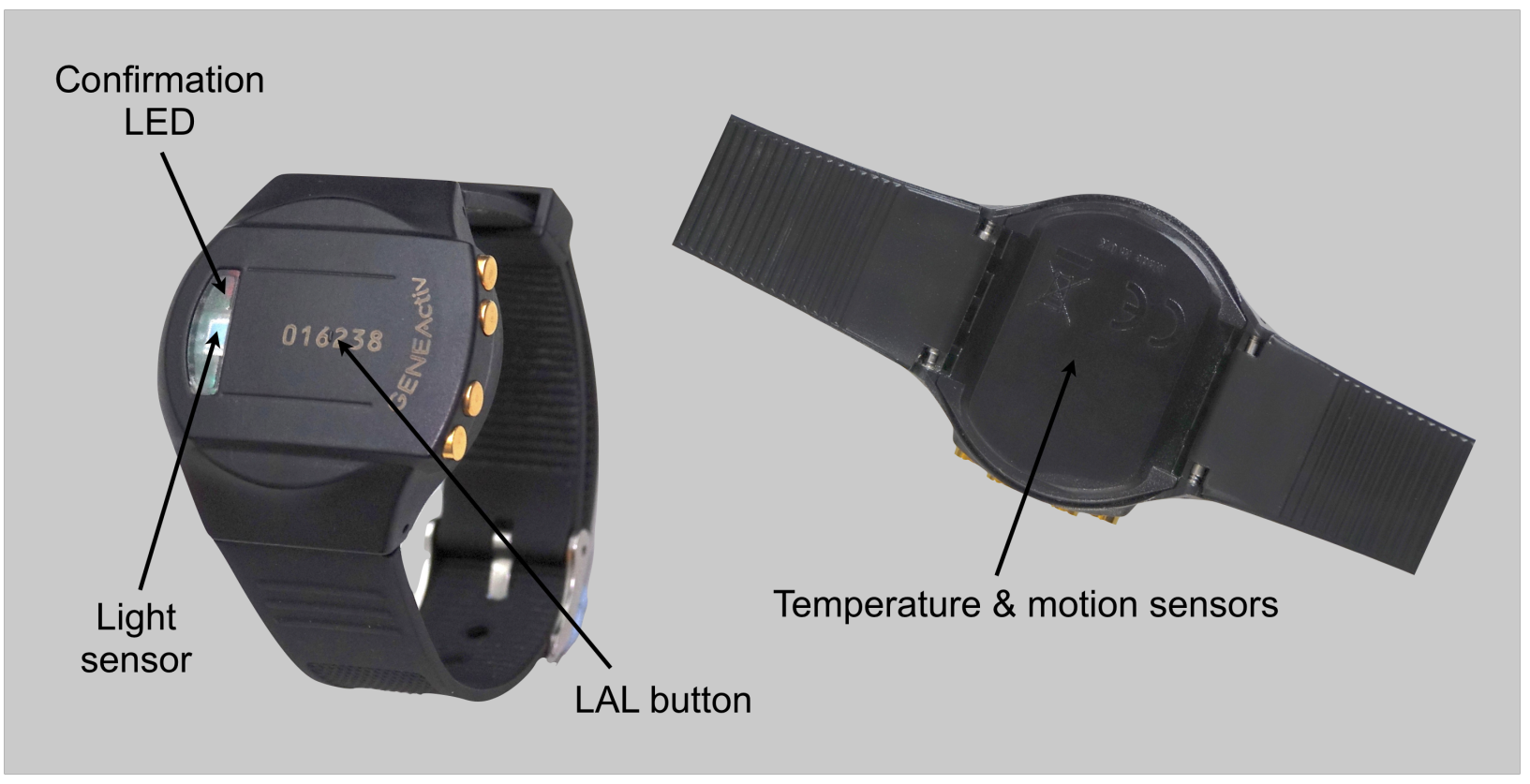

Figure 1: The wrist-mounted electronic logging device, the GeneActiv activity monitor from a front view (left) and a rear view (right) highlighting the sensors, LED and button.

presses had to be completed within a 10-second window, with button presses outside of this window then disregarded for the next 50 seconds (to again allow the participant to forget about the reduction in contact lens comfort). If lens awareness was still present after the 1-minute period, the participant was instructed to press the LAL button again and to continue doing so (every minute) until the awareness settled. Using this approach, each LAL event highlighted a minute period during contact lens wear, where the patient had experienced ocular discomfort and were classed as discrete events even when they occured in consecutive minute periods. Once data collection had been completed over the three study days, the device was returned to the clinic and placed into its docking station, with the internal data downloaded via a USB interface. These data were then imported into custom MATLAB software (MathWorks Inc., Natick, NC.) where the outputs from the various sensors and button could be visualised and the validity of these data confirmed (Figure 2). The software allowed the investigator to confirm the time of lens application and removal, with the software then calculating (i) time of lens application / removal (ii) the total number of LAL events, and (iii) the number of LAL events per hour.

\subsection{Clinical study}

This was a prospective, controlled, randomised, participant-masked study where contact lens awareness was monitored during wear of two different study contact lens types. This study was comprised of two clinical visits separated by three lens wearing days, during which time participants recorded their lens awareness with the LAL. The aim of this work was to investigate if the LAL was able to detect differences 

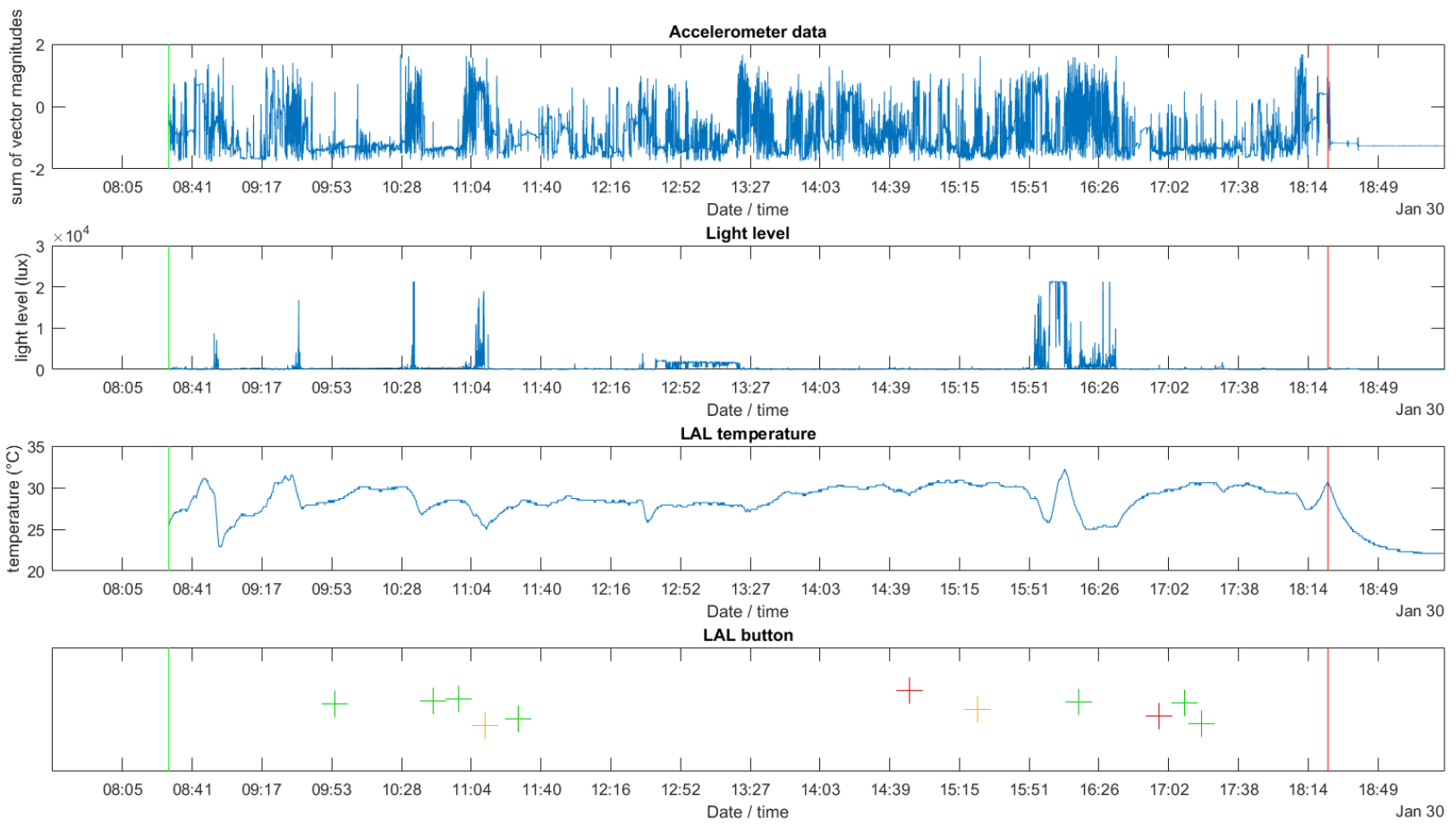

Figure 2: A typical screenshot of the LAL analysis software from study day 3, highlighting the output of the accelerometer, light and temperature sensor, in addition to the LAL button events (green line = lenses applied, red line $=$ lenses removed, green cross $=$ single LAL event, orange cross $=$ double LAL event, red cross $=$ triple LAL event). 
in wearer awareness between two brands of contact lenses (Lens A: senofilcon A - Acuvue Oasys, Johnson \& Johnson Vision Inc. and Lens B: balafilcon A - PureVision, Bausch \& Lomb Inc.), that have been shown to demonstrate differences in end of day discomfort [14, 15]. Participants wore both lens types, as a matched pair, on day 1 and day 2 of the study (randomised order). Additionally, to ascertain if participants could provide more detailed information about the intensity of any lens awareness, participants used a more complex multiple-click reporting system on the third lens wearing day (only lens B worn). Lenses were worn on a daily wear, daily disposable basis (i.e. removed at night and discarded). This study was controlled by cross-comparison, with bias minimised by masking the participants to the lenses worn on each of the LAL days (by over-labelling of the lens blister packaging). Prior to starting the study, participants were evaluated on the CLDEQ-8 questionnaire and only participants with contact lens dry eye (CLDE) or marginal CLDE (as defined by Young et al.[20]) were eligible for this work. In total 30 participants were recruited.

All participants provided written informed consent before inclusion in the study. The study was conducted in accordance with the principles of the Declaration of Helsinki and The University of Manchester Research Ethics Committee provided ethical approval. Individuals with a history of ocular/systemic disorders that would normally contraindicate contact lens wear, a history of ocular surgery, who were using topical ophthalmic medication, who had corneal distortion, who were pregnant or breastfeeding, who had a history of anaphylaxis or severe allergic reaction or any infectious or immunosupressive disease that would pose a risk to study personnel and users of either of the study lens types were excluded from participating in the study. Each participant was required to attend two clinic visits - an initial visit and a final visit (4-7 days after initial visit). At the initial visit and prior to contact lens application, subjective ocular comfort was assessed using a 0-100 visual analogue scale (VAS). Refraction, visual acuity assessment and slit lamp biomicroscopy were then performed. Both study lens types were applied in sequence and their fit evaluated. The participant was then instructed on how to use the LAL (described in Section 2.1) and was asked to use the device on days where they were performing similar activities (e.g. normal work days or similar non-working days). The participant was then issued with the LAL and the three pairs of the lenses (two pairs of Lens B and one pair of Lens A to their own refractive prescription) and asked to return for the final visit, after completion of the three days of lens wear / LAL usage. On the first day of LAL use, the participants applied a pair of study lens (either Lens A or Lens B). Lens awareness events associated with ocular discomfort were then recorded through the day using a simple single click protocol (i.e. a single click each time the participant had lens awareness). Participants were instructed to use the study lenses for as long as they were comfortable, with a target of at least 8 hours of study lens wear where possible. Immediately following lens removal, subjective comfort was assessed using a 0-100 VAS grading scale (relating to comfort experienced during contact lens wear). On the second day of LAL use, participants again used the LAL to record lens awareness events with the other lens type (single click protocol) and subjective comfort with the 0-100 VAS grading scale. On the third day of LAL use, all participants applied a pair of Lens B and 
used the multiple click protocol. Following lens removal, subjective comfort was assessed using a 0-100 VAS grading scale (relating to the comfort during study contact lens wear). At the final clinic visit, participants were questioned on the ease of use of the LAL (using a five point Likert scale) and their use of the LAL device was reviewed to confirm it was used as instructed. The study participant was also asked to review the LAL data and to confirm whether it was representative of their use of the device. Refraction, visual acuity assessment and slit lamp biomicroscopy were then performed and the participant was exited from the study.

\subsection{Power analysis}

In previous work, the mean number of 'lens awareness events' was 1.2 per hour on the first day of use and 1.4 per hour on the second day of use [13]. Given this, it was proposed that a difference of 0.5 lens awareness events per hour may be of clinical significance. A power analysis indicated that to detect this magnitude of difference 26 participants would provide 0.80 statistical power, given a standard deviation of intra-participant day-to-day differences of 0.88 (from the previous LAL study [13]) and an alpha of 0.05 . Thirty participants were therefore recruited to allow for any discontinuations.

\subsection{Statistics}

The main outcome measure in this work was lens awareness events per hour. The principal hypothesis to be tested in this work was that lens awareness as assessed with the LAL was equivalent for the two study lens types (Lens A and Lens B). Period of study lens wear, lens awareness events per day, lens awareness events per hour and 0-100 VAS comfort data were compared between the two study lens types and between the study days (days 1 and 2), using paired t-tests. To allow the LAL data to be summarised over the daily lens wearing period for the two study lens types, the data were normalised with respect to wear time (i.e. the contact lens wearing time for each set of LAL data was split into 10 evenly spaced periods and the number of LAL events within each period recorded). To compare the LAL profile for the two lens types, the difference in LAL events for each normalised time period were plotted, with a lack of overlap of the $95 \%$ confidence intervals with zero indicating a significant difference between lens types. To investigate the relationship between 0-100 VAS subjective comfort scores and LAL metrics, Pearson correlation coefficients were calculated. A p-value of less than 0.05 was considered statistically significant. All data were analysed using JMP 14, Version 14.3 (SAS Institute Inc. Cary, NC, USA).

\section{Results}

\subsection{Single click protocol - study day 1832}

Table 1 details the participant demographics and CLDEQ-8 questionnaire scores for the participants habitual contact lenses. Of the 30 participants recruited, 29 participants completed the clinical study, with 
one participant lost to follow-up. On reviewing the LAL data, the data from three participants was discarded from analysis due to non-compliance with the LAL device (e.g. removing the LAL device during contact lens wear or otherwise not following the study instructions). The LAL analysis therefore included data from 26 participants. The study contact lens wearing period did not differ significantly between the two study days $(\mathrm{p}=0.10$; Day 1: $13.2 \pm 1.6$ hours / Day 2: $12.7 \pm 2.0$ hours) or between the two study lens types $(\mathrm{p}=0.31$; Lens A: $13.2 \pm 1.9$ hours / Lens B: $12.8 \pm 1.8$ hours $)$. The mean contact lens wearing time was $13.0 \pm 1.8$ hours (range: 9.7 to 16.0 hours). On reviewing the LAL data at the follow-up visit, all subjects confirmed that the data captured were representative of their lens wearing experience

\begin{tabular}{cccc}
\hline Parameter & Females & Males & Overall \\
\hline Number of participants & 18 & 8 & 26 \\
Age & $31.0 \pm 9.5(19$ to 49$)$ & $22.7 \pm 4.3(19$ to 31$)$ & $28.7 \pm 9.1(19$ to 49$)$ \\
Best Sphere (D) & $-2.77 \pm 1.28(-6.00$ to -1.00$)$ & $-2.34 \pm 1.03(-3.50$ to -1.00$)$ & $-2.65 \pm 1.22(-6.00$ to -1.00$)$ \\
Cylinder (D) & $-0.31 \pm 0.24(-0.75$ to 0.00$)$ & $-0.27 \pm 0.23(-0.75$ to 0.00$)$ & $-0.30 \pm 0.24(-0.75$ to 0.0$)$ \\
CLDEQ-8 (habitual lenses) & $13.9 \pm 4.5(8$ to 27$)$ & $12.8 \pm 4.8(8$ to 20$)$ & $13.6 \pm 4.6(8$ to 27$)$ \\
\hline
\end{tabular}

Table 1: Participant demographics and CLDEQ-8 questionnaire scores relating to the participants habitual contact lenses (mean \pm standard deviation with range in parentheses).

The LAL events per day did not differ significantly between the two study days (Day 1: $17.3 \pm 17.4$ events, Day 2: $15.8 \pm 16.6$ events; $\mathrm{p}=0.72)$, but was significantly higher for Lens B $(21.6 \pm 21.0$ events $)$ compared to the Lens A $(11.6 \pm 9.2$ events ; $\mathrm{p}=0.0063$; Figure $3 \mathrm{~A})$. A similar trend was observed for the mean LAL events per hour data which did not differ significantly between the two study days (Day 1: 1.4 \pm 1.6 events/hour, Day 2: $1.3 \pm 1.6$ events/hour; $\mathrm{p}=0.87$ ), but was significantly higher for Lens $\mathrm{B} 1.8 \pm$ 2.1 events/hour) compared to Lens A $(0.9 \pm 0.7$ events/hour; $\mathrm{p}=0.01$; Figure $3 \mathrm{~B})$. The distribution of LAL events through the daily lens wearing cycle is shown in Figure 3C. The daily LAL event profile highlighted increased lens awareness following lens application (thought to be related to contact lens settling) and prior to lens removal (thought to be related to end of day discomfort). Figure 3D details the LAL event profile by study lens type, highlighting minimal initial lens awareness for Lens A in comparison with Lens B, although both lens types appeared to show an increase in lens awareness towards the end of the lens wearing period. Analysis of difference in the LAL events profile between the two lens types (Figure 3E) highlighted a significant difference between the lens types (i.e. where the $95 \%$ confidence intervals of the mean difference do not cross zero) at 10\%,20\%,30\%, 50\% and $80 \%$ of the total daily lens wearing period. This highlights a greater difference in lens awareness between the lens types earlier in the lens wearing period, with a less marked difference in lens awareness as the day progressed (as highlighted by the trend for the LAL data to generally converge through the lens wearing period for the two lens types, shown in Figure 3D).

Subjective comfort scores (0-100 VAS grading scale) for the two study lens types are shown in Table 2. Mean 0-100 VAS comfort scores were highest following lens application, followed by the overall score, with 
A

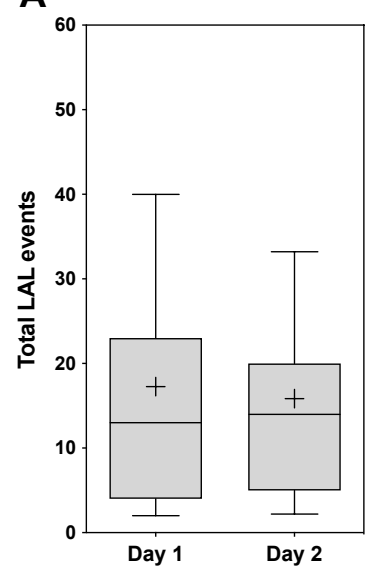

B

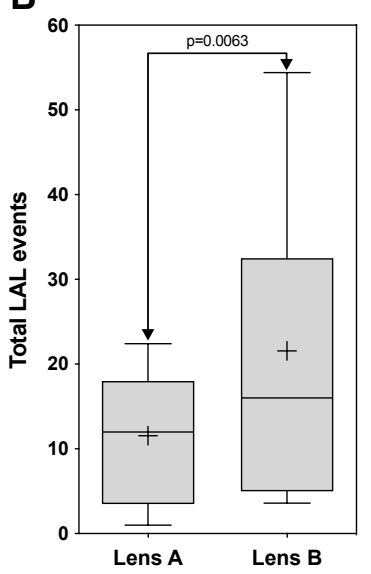

C

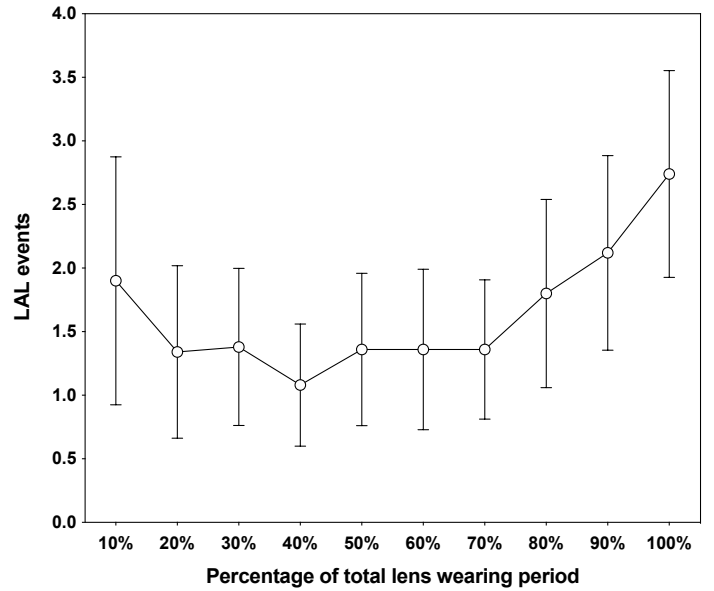

E

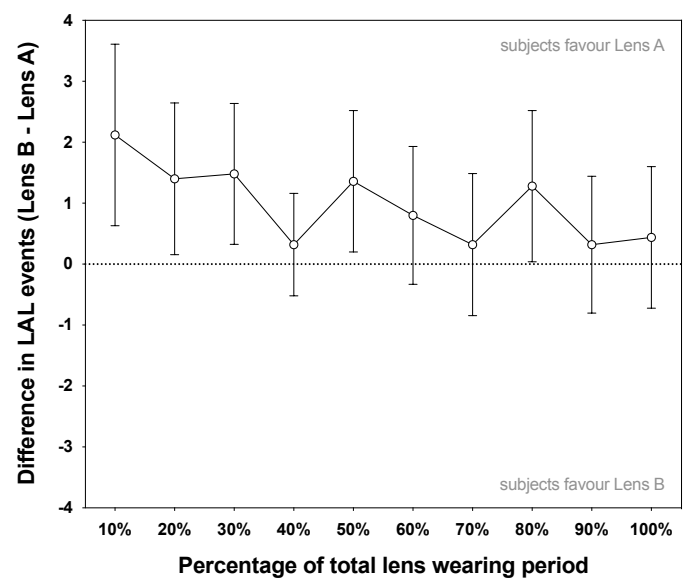

Figure 3: The total number of LAL events (A) and LAL events per hour (B) for the two study lens types. The lens awareness profile through the lens wearing period $(\mathrm{C})$ and by lens types (D). The difference in LAL response between the two study lens types (E). For the box and whisker plots, the cross indicates the mean, the line the median, the box the 25th and 75th centiles and the whiskers the max and min values. For the line plots, the error bars indicating the $95 \%$ confidence intervals. All data in this Figure relate to the single click protocol during study day 1 and 2 only. 
the lowest mean comfort score recorded immediately prior to lens removal. Lens B showed a consistently lower mean comfort score (typically around 10 points lower on the 100 point scale), although the difference was only statistically significant for the initial comfort $(\mathrm{p}=0.03)$ and overall comfort $(\mathrm{p}=0.04)$; and did not reach significance for the end of day comfort $(\mathrm{p}=0.10)$.

\begin{tabular}{cccc}
\hline Lens type & Comfort on lens application & Overall comfort & Comfort prior to lens removal \\
\hline Lens A & $90.9 \pm 8.7$ & $78.0 \pm 12.9$ & $64.4 \pm 19.0$ \\
Lens B & $81.6 \pm 17.7$ & $69.2 \pm 19.4$ & $54.2 \pm 20.5$ \\
\hline P-value & $\mathrm{P}=0.03$ & $\mathrm{P}=0.04$ & $\mathrm{P}=0.10$
\end{tabular}

Table 2: 0-100 VAS comfort scores captured at the end of the study day, immediately prior to lens removal, for the two study lens types (mean \pm standard deviation).

\subsection{Multiple click protocol - study day 3}

For data captured using the multiple click protocol, a significant difference was observed between the mean number of single, double and triple clicks $(\mathrm{p}=0.0001)$. The mean number of clicks per day was highest for the single click (11.0 per day), followed by the double click protocol (6.9 per day) and lowest for the triple clicks (3.1 per day). Figure 4A highlights the LAL profile for the multiple click protocol. There was generally a low incidence of severe lens awareness (triple clicks) except at the end of the lens wearing day where an increased incidence was observed. To summarise these findings in a single profile, each LAL event was weighted by the severity of the symptoms (i.e. single clicks $=1$, double clicks $=2$ and triple clicks $=3$ ), as shown in Figure 4B. This highlighted the key periods of increased lens awareness (in the case of Lens B this was again following lens application and prior to lens removal). This lens awareness was then summarised into a single metric (the lens awareness index - LAI), by summing the average weighted scores across the lens wearing period (mean LAI for Lens B $=32.1$ ).

To investigate the relationship between overall comfort scores (0-100 VAS) and (i) LAL events (day one and two), and (ii) LAI (day three) a correlation analysis was performed, as shown in Figure 5. A moderate negative correlation $(\mathrm{r}=-0.76)$ was observed between overall comfort and daily LAL events (day one and two), which reached statistical significance $(\mathrm{p}<0.0001)$. A moderate negative correlation $(\mathrm{r}=-0.79)$ was also observed between overall comfort and LAI (day three), which reached statistical significance $(\mathrm{p}<0.0001)$.

At the final clinical visit the participant was asked to reflect on the use of the LAL device. Figure 6 highlights the participants agreement with the statements relating to ease to remember to log lens awareness events, and ease of use of the LAL for the single and multiple click protocol. The participants were generally in agreement with the statements around ease of use and remembering to use the device to log awareness, with less than $15 \%$ of participants giving negative responses to the three questions. Where negative responses were given this generally related to the button on the LAL device, which some participants found required 
A

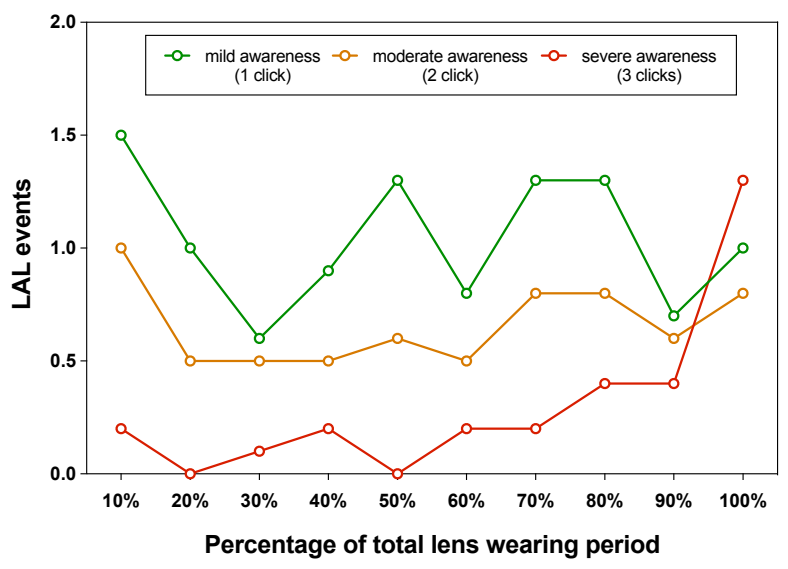

B

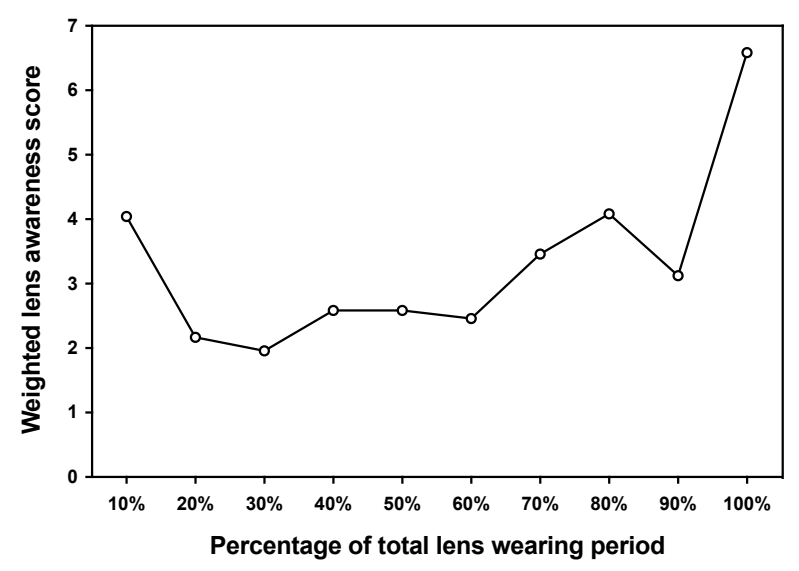

Figure 4: The lens awareness profile (A) and the weighted lens awareness profile (B) through the lens wearing period for the multiple click protocol (green line $=$ mild lens awareness $/$ single click, orange line $=$ moderate lens awareness $/$ double click and red line $=$ severe lens awareness / triple click).
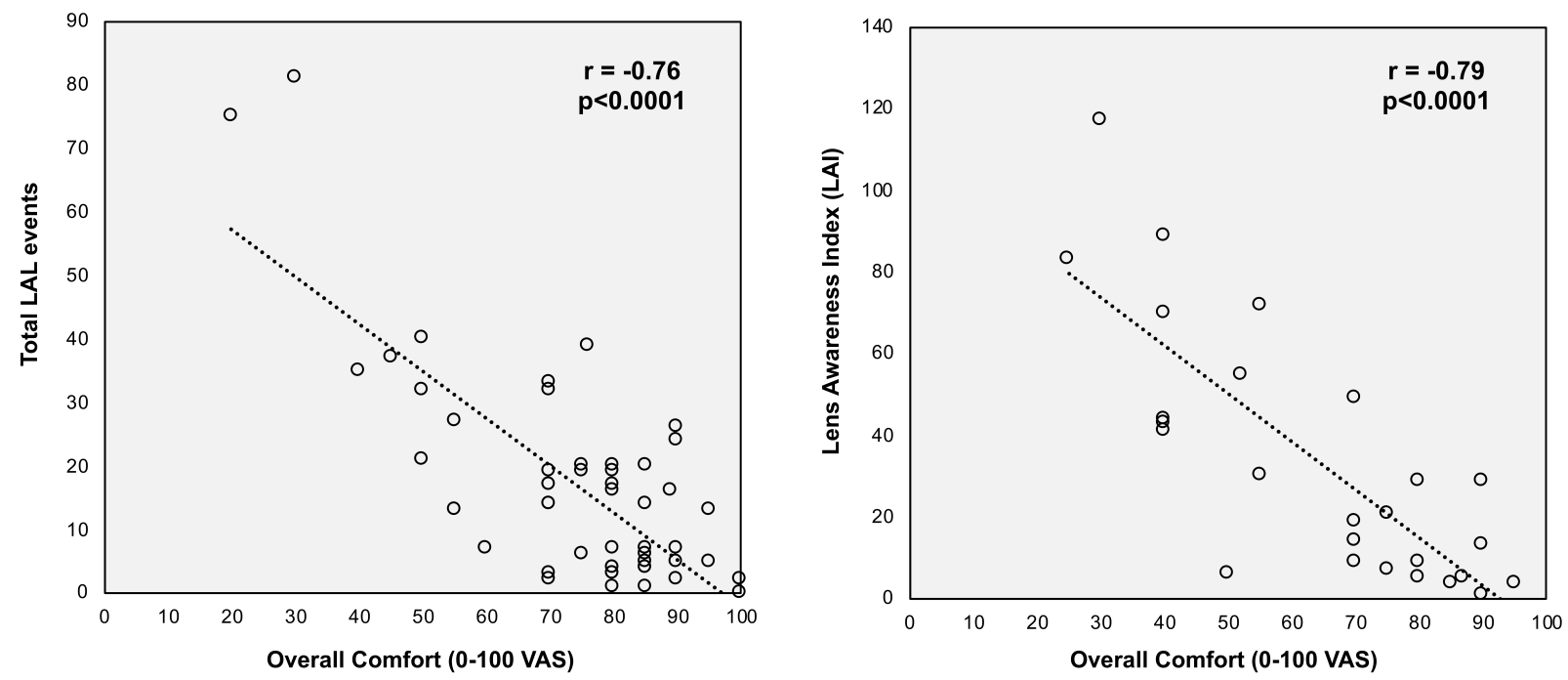

Figure 5: Correlation between total LAL events (study day 1 and 2) and subjective comfort scores (left) and LAI (study day 3) and subjective comfort scores (right). 


\section{Percentage of total responses}

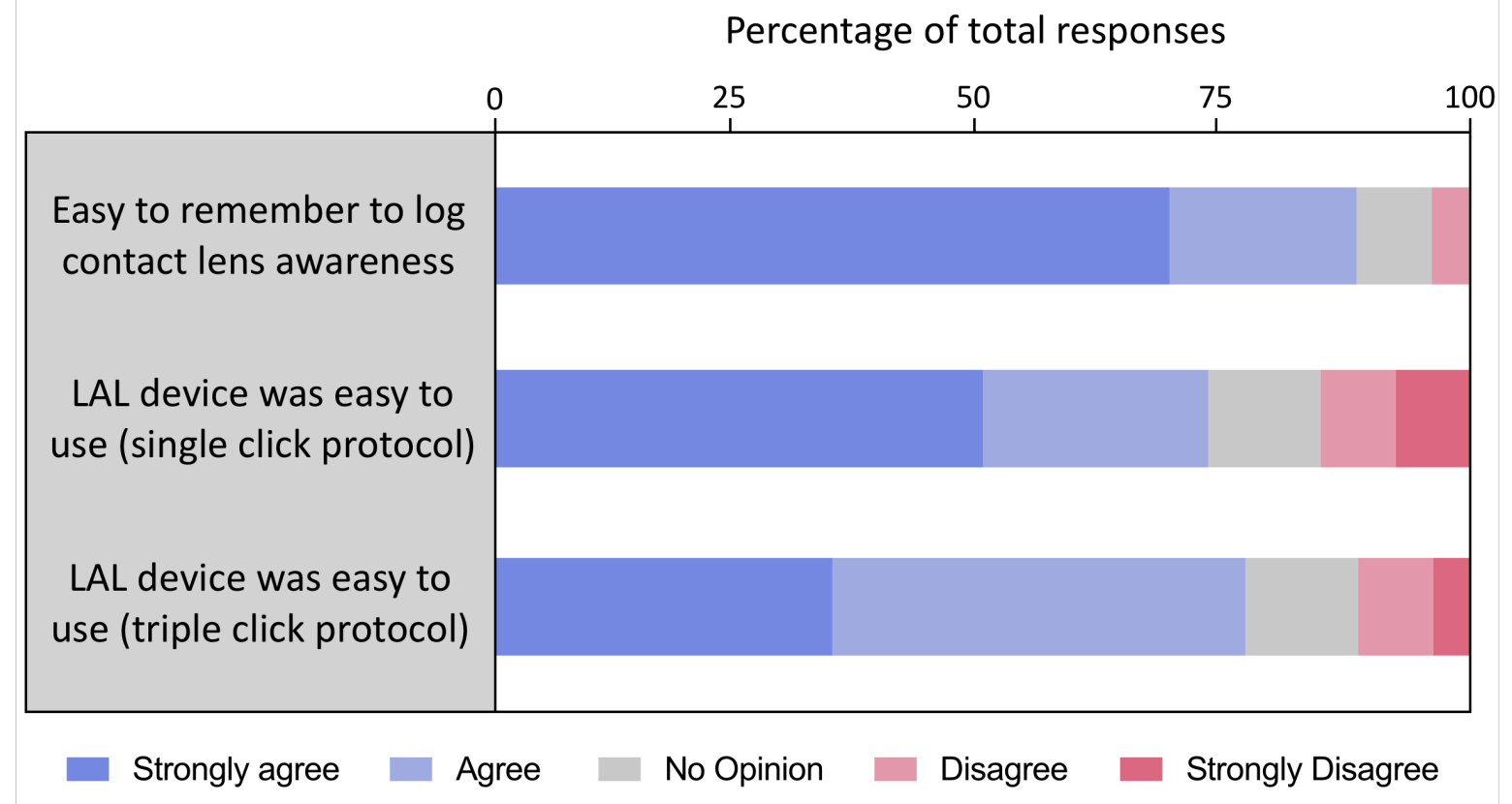

Figure 6: Horizontal stacked bar graph with the responses to three questions relating to the ease of the use of the LAL device,

with the Likert responses detailed below.

a firm press to confirm a LAL event (the button is designed in this manner to avoid accidental activation during wear).

\section{Discussion}

Although contact lenses are worn on the most highly innervated tissue of the human body [1], the combination of soft biocompatible materials and high precision manufacture allow contact lenses to be worn with a high level of comfort for the majority of patients. However, it is thought that around $50 \%$ of the contact lens wearing population still experience symptoms of dryness and/or discomfort at least occasionally during lens wear $[21,22]$. This reduction in comfort is typically not uniform through the wearing period, but often intermittent, with ocular irritation occurring sporadically through the wearing cycle [13]. Scoring of this type of transient and intermittent irritation is challenging and requires the patient to average out the lens wearing experience through the period of wear. The LAL device has previously been used to monitor ocular awareness, highlighting that symptomatic contact lens wearers had a greater number of lens awareness events and more frequent symptoms later in the lens wearing period, in comparison with asymptomatic contact lens wearers [13]. Given that symptomatic contact lens wearers are more prone to contact lens discontinuation [23] and present a significant clinical management challenge, it was decided that a symptomatic participant group would be the focus of this work. 
The participant population was typical of the general contact lens wearing population, with a female bias, moderately myopic prescription range and an average age of around 30 years [24]. The use of the validated CLDEQ-8 questionnaire [25] allowed participants with contact lens related dryness symptoms to be recruited into the study, with the questionnaire scores suggesting a similar population to the symptomatic participant group in the previous LAL clinical study [13]. The daily lens wear period in this study was similar over the three study days and typical of that recorded in the literature for daily soft contact lens wear [26, 22, 21]. The subjective comfort grading scores (0-100 VAS scale) observed in this study confirmed the expected difference between lens types, with the Lens A on average scoring around 10 points higher on the 100 point VAS scale. By studying these lens types, it was therefore anticipated that these differences would be elucidated by the LAL testing methodology. The number of LAL events per day and the mean number of LAL events per hour were both shown to be significantly higher for Lens B, than for Lens A, highlighting the ability of the system to differentiate between lens types with different comfort characteristics. In agreement with the previous LAL study [13], the number of LAL events recorded was similar between study day 1 and day 2, indicating that a significant learning or fatigue effect was not evident during use of the LAL device. As was observed in the previous LAL study, the distribution of LAL events was not uniform through the lens wearing cycle, but was typically elevated after lens application and towards the end of the lens wearing cycle. This initial lens awareness is often termed lens settling and is thought to relate to tear film disruption, contact lens hydration changes and resultant dimensional/lens movement alterations after a contact lens is applied to the ocular surface [27], in addition to possible sensory adaptation to lens wear [28]. This process of lens settling is generally reported to last for up to 30 minutes [27], which coincides with the initial peak in lens awareness events observed for the study lenses. It is worth noting that the initial awareness due to lens settling is more marked in Lens B, suggesting that factors such as its higher modulus [29], higher surface friction [30], less wettable surface [31,32] and/or rounded edges [14] may result in a lens that is less able to quickly settle on the ocular surface. This difference in the degree of lens awareness between the two study lens types, becomes less apparent later in the wearing cycle, with both lens types showing an increase in awareness in the last few hours of the daily lens wearing period. This increase in lens awareness typically observed later in the daily lens wearing period is often term 'end-of-day (EOD) contact lens discomfort', the cause of which is not fully understood [33]. Previous work has indicated that EOD discomfort associated with contact lens wear does not appear to be primarily related to an aging/degrading lens or to the time of day, but rather determined by the length of time lenses are in contact with the eye [34]. The findings of this study also highlight that the factors influencing EOD discomfort are likely to be different from those influencing initial settling, with the two lens types having very different mechanical, surface and lens design characteristics, but yet showing a similar trend for EOD discomfort.

An obvious limitation of the LAL device (in study day 1 and day 2) was that the single click protocol gave no weighting as to the severity of the lens awareness, rather allocating a binary event at points of 
lens awareness through the wearing cycle. In an attempt to address this short coming, a multiple click protocol was evaluated on the third lens wearing study day. Here the participant logged either a single, double or triple click (mild, moderate or severe awareness respectively) depending on the severity of the lens awareness. This approach appeared well accepted by the study participants, who reported similar ease of use to that recorded for the single use protocol. The single-click (mild awareness) was generally the most frequently logged event, followed by the double-click (moderate awareness) and least commonly used was the triple-click (severe awareness). The profile of LAL events through the day followed a similar profile to that recorded previously, with increased awareness at the beginning and end of the lens wearing cycle. The triple click events were typically low in frequency (less than 1 event per 2 hours of lens wear on average), although towards the end of the lens wearing period the frequency increased dramatically $(>1$ event per hour on average), indicating an increase in the severity of lens awareness events later in the wearing cycle. This increase in the severity of symptoms, has been previously observed later in the lens wearing cycle using both conventional grading and text messaging systems $[35,8]$. By weighting the LAL events according to their severity, a more meaningful profile through the day was generated, which is thought to relate to how bothersome the contact lens had been, in addition to a single metric (LAI) which summarises the degree of awareness into a single value. The weighted profile and LAI metric are likely to be useful in future comparative clinical studies to highlight key differences in the lens awareness experience between different lens types and/or care systems.

The use of an electronic event logger to monitor comfort-related awareness events has allowed the monitoring of such unwanted episodes and is likely to be a useful tool in better characterising the clinical performance of contact lens designs, materials and care systems. This is a key area for improved understanding, as it has been clearly shown that poor levels of comfort during lens wear is a leading cause of discontinuation from lens wear [21], limiting the size of the contact lens market. More broadly, the LAL device could be used to characterise episodes of ocular discomfort in a range of optometric and ophthalmogical conditions, such as dry eye disease, ocular allergy, the effectiveness of ocular analgesics or for monitoring recovery from ocular surgery. In addition, visual quality is a key property of an optical device and whilst single vision spectacles and contact lenses generally provide high quality and stable vision; multifocal and toric corrections are frequently associated with variable vision [36, 37, 38]. The LAL device could therefore also be used to monitor episodes of awareness associated with sub-optimal vision.

A further useful element of the LAL device is that whilst it is recording episodes of comfort/vision related awareness, it is also logging the output from a range of internal sensors. In this study, the intention of monitoring the light, temperature and accelerometer data was solely to ensure the device was worn by the participant as intended, by monitoring for change in the output from these three sensors. As the temperature sensor was monitoring body temperature (i.e. not environmental temperature), the light sensor could be obscured by clothing and the accelorometer data was not calibrated for physical activity, further 
analysis was not possible in this study. However in future studies, the wear of the LAL device and setup of the sensors could be modified to investigate the relationship between episodes of lens awareness and the lens wearing environment, such as the light level (via the photo-diode sensor) with the performance of a multifocal contact lens or time spent outdoors (via the temperature and photo-diode sensor) with contact lens comfort. In addition, this awareness data could be linked with externally captured data such as activities being undertaken (using a detailed diary recording) or factors such as blink rate (using a head mounted eye tracker). Such an approach is likely to provide useful information on the factors which influence clinical performance. A possible future implementation of the LAL device could also allow it to capture more specific information about the symptoms experienced, either via speech input or a small digital touch screen display. However, by adding greater capability to the data capture system, there is a potential that this may interfere with the simplistic and minimally invasive nature of data capture present with the current LAL approach. Where additional complexity is added to the data capture system, the additional information gained with such an approach should be carefully weighed up against the increased burden on the participant.

\section{Conclusion}

In conclusion, the LAL device was able to differentiate between the two lens types, with Lens $\mathrm{B}$, as expected, resulting in a greater number of LAL events per study day than Lens A. The distribution of LAL events through the lens wearing period also differed between the lens types, with Lens B resulting in more awareness, particularly in the first half of the lens wearing period. The multiple-click LAL protocol highlighted that discomfort-related lens awareness appeared to increase not only in frequency, but also in intensity towards the end of the lens wearing cycle. The LAL device was well accepted by the participants using both the single and multiple-click protocol, and the additional logging capabilities of the wrist-mounted LAL device allowed exclusion of non-compliant participant data. The ability of the LAL to track lens awareness suggests it is likely to be a key tool in furthering understanding of ocular discomfort.

\section{Acknowledgements}

The study was funded by CooperVision Inc.

\section{References}

[1] C. Belmonte, J. Gallar, Corneal Nocicrptors, in: C. Belmonte, F. Cervero (Eds.), Neurobiology of Nociceptors, Oxford University Press, Oxford, 1996, p. 146.

[2] C. Belmonte, M. Carmen Acosta, J. Merayo-Lloves, J. Gallar, What Causes Eye Pain?, Curr Ophthalmol Rep 3 (2015) 111-121.

[3] F. Amparo, D. A. Schaumberg, R. Dana, Comparison of Two Questionnaires for Dry Eye Symptom Assessment, Ophthalmology 122 (2015) 1498-1503. 
[4] D. A. Schaumberg, A. Gulati, W. D. Mathers, T. Clinch, M. A. Lemp, J. D. Nelson, G. N. Foulks, R. Dana, Development and Validation of a Short Global Dry Eye Symptom Index, Ocul Surf 5 (2007) 50-57.

[5] K. E. Kip, F. Cohen, S. R. Cole, K. R. Wilhelmus, D. L. Patrick, R. Blair, R. W. Beck, Recall bias in a prospective cohort study of acute time-varying exposures, J Clin Epidemiol 54 (2001) 482-487.

[6] J. E. Broderick, A. A. Stone, P. Calvanese, J. E. Schwartz, D. C. Turk, Recalled Pain Ratings: A Complex and Poorly Defined Task, J Pain 7 (2006) 142-149.

[7] K. Dumbleton, N. Keir, A. Moezzi, Y. Feng, L. Jones, D. Fonn, Objective and subjective responses in patients refitted to daily-wear silicone hydrogel contact lenses, Optom Vis Sci 83 (2006) 758-768.

[8] M. Navascues-Cornago, P. B. Morgan, C. Maldonado-Codina, Effect of three interventions on contact lens comfort in symptomatic wearers: A randomized clinical trial, PLoS ONE 10 (2015) e0135323.

[9] C. A. Woods, K. Dumbleton, L. Jones, D. Fonn, Patient use of smartphones to communicate subjective data in clinical trials, Optom Vis Sci 88 (2011) 290-294.

[10] A. A. Stone, S. Shiffman, J. E. Schwartz, J. E. Broderick, M. R. Hufford, Patient non-compliance with paper diaries, BMJ 324 (2002) 1193-1194.

[11] A. K. Yetisen, J. L. Martinez-Hurtado, B. Ünal, A. Khademhosseini, H. Butt, Wearables in Medicine, Adv Mater 30 (2018) 1706910 .

[12] Guk, Han, Lim, Jeong, Kang, Lim, Jung, Evolution of Wearable Devices with Real-Time Disease Monitoring for Personalized Healthcare, Nanomaterials 9 (2019) 813.

[13] M. L. Read, P. B. Morgan, C. Maldonado-Codina, A wearable device to monitor ocular comfort, Cont Lens Anterior Eye 42 (2019) 569-574.

[14] C. Maissa, M. Guillon, R. J. Garofalo, Contact Lens-Induced Circumlimbal Staining in Silicone Hydrogel Contact Lenses Worn on a Daily Wear Basis, Eye Contact Lens 38 (2012) 16-26.

[15] J. Diec, V. E. Evans, D. Tilia, T. Naduvilath, B. A. Holden, P. L. De La Jara, Comparison of ocular comfort, vision, and SICS during silicone hydrogel contact lens daily wear, Eye Contact Lens 38 (2012) 2-6.

[16] S. E. Jones, V. T. van Hees, D. R. Mazzotti, P. Marques-Vidal, S. Sabia, A. van der Spek, H. S. Dashti, J. Engmann, D. Kocevska, J. Tyrrell, R. N. Beaumont, M. Hillsdon, K. S. Ruth, M. A. Tuke, H. Yaghootkar, S. A. Sharp, Y. Ji, J. W. Harrison, R. M. Freathy, A. Murray, A. I. Luik, N. Amin, J. M. Lane, R. Saxena, M. K. Rutter, H. Tiemeier, Z. Kutalik, M. Kumari, T. M. Frayling, M. N. Weedon, P. R. Gehrman, A. R. Wood, Genetic studies of accelerometer-based sleep measures yield new insights into human sleep behaviour., Nat. Commun. 10 (2019) 1585.

[17] T. G. Pavey, S. R. Gomersall, B. K. Clark, W. J. Brown, The validity of the GENEActiv wrist-worn accelerometer for measuring adult sedentary time in free living, J Sci Med Sport 19 (2016) 395-399.

[18] E. G. Landis, V. Yang, D. M. Brown, M. T. Pardue, S. A. Read, Dim light exposure and myopia in children, Invest Ophth Vis Sci 59 (2018) 4804-4811.

[19] A. K. Rao, Wearable Sensor Technology to Measure Physical Activity (PA) in the Elderly, Curr Geriatr Rep 8 (2019) $55-66$.

[20] G. Young, R. L. Chalmers, L. Napier, C. Hunt, J. Kern, Characterizing contact lens-related dryness symptoms in a cross-section of UK soft lens wearers, Cont Lens Anterior Eye 34 (2011) 64-70.

[21] N. Pritchard, D. Fonn, D. Brazeau, Discontinuation of contact lens wear: A survey, Int Contact Lens Clin 26 (1999) $157-162$.

[22] K. Richdale, L. T. Sinnott, E. Skadahl, J. J. Nichols, Frequency of and factors associated with contact lens dissatisfaction and discontinuation, Cornea 26 (2007) 168-174.

[23] K. Dumbleton, C. A. Woods, L. W. Jones, D. Fonn, The Impact of Contemporary Contact Lenses on Contact Lens Discontinuation, Eye Contact Lens 39 (2013) 92-98. 
[24] P. Morgan, C. Woods, I. Tranoudis, N. Efron, L. Jones, W. Aighamdi, V. Nair, N. Merchan, M. Teufl, C. Grupcheva, D. Jones, M. Beeler-Kaupke, W. Lan, P. . Qi, K. O. Tan, J. Krasnanska, H. Pult, O. Ravn, J. SantodomingoRubido, P. Tast, F. Malet, A. Plakitsi, M. Vegh, N. Erdinest, P. Chande, Montani Giancarlo, M. Itoi, J. Bendoriene, R. Ramos Gomez, R. Pintor, E. Van Der Worp, W. Lam, A. E. Ystenae, J. Romualdez, C. Abesamis-Dichoso, J. M. Gonzalez-Meijome, V. Belousov, D. Sim, M. Stabuc Silih, P. Gierow, A. Lindskoog Pettersson, J. Hsiao, J. Nichols, International Contact Lens Prescribing in 2018, Contact Lens Spectrum 34 (2019) $26-32$.

[25] R. L. Chalmers, C. G. Begley, K. Moody, S. B. Hickson-Curran, Contact Lens Dry Eye Questionnaire-8 (CLDEQ-8) and Opinion of Contact Lens Performance, Optom Vis Sci 89 (2012) 1435-1442.

[26] J. J. Walline, K. O. Lorenz, J. J. Nichols, Long-term contact lens wear of children and teens, Eye Contact Lens 39 (2013) $283-289$.

[27] J. J. Nichols, P. E. King-Smith, The Impact of Hydrogel Lens Settling on the Thickness of the Tears and Contact Lens, Invest Ophth Vis Sci 45 (2004) 2549.

[28] P. Situ, T. L. Simpson, L. W. Jones, D. Fonn, Effects of silicone hydrogel contact lens wear on ocular surface sensitivity to tactile, pneumatic mechanical, and chemical stimulation, Invest Ophth Vis Sci 51 (2010) 6111-6117.

[29] E. Kim, M. Saha, K. Ehrmann, Mechanical Properties of Contact Lens Materials, Eye Contact Lens 44 (2018) S148-S156.

[30] M. Roba, E. G. Duncan, G. A. Hill, N. D. Spencer, S. G. P. Tosatti, Friction Measurements on Contact Lenses in Their Operating Environment, Tribol Lett 44 (2011) 387-397.

[31] M. L. Read, P. B. Morgan, J. M. Kelly, C. Maldonado-Codina, Dynamic Contact Angle Analysis of Silicone Hydrogel Contact Lenses., J Biomater Appl 26 (2010) 85-99.

[32] M. L. Read, P. B. Morgan, Measurement errors related to contact angle analysis of hydrogel and silicone hydrogel contact lenses, J Biomed Mater Res A 91 (2009) 662-668.

[33] J. J. Nichols, M. D. Willcox, A. J. Bron, C. Belmonte, J. B. Ciolino, J. P. Craig, M. Dogru, G. N. Foulks, L. Jones, J. D. Nelson, K. K. Nichols, C. Purslow, D. A. Schaumberg, F. Stapleton, D. A. Sullivan, The TFOS International Workshop on Contact Lens Discomfort: Executive summary, Invest Ophth Vis Sci 54 (2013) 7-13.

[34] E. Papas, D. Tilia, J. McNally, P. L. De La Jara, Ocular discomfort responses after short periods of contact lens wear, Optom Vis Sci 92 (2015) 665-670.

[35] J. Wolffsohn, L. Hall, S. Mroczkowska, O. A. Hunt, P. Bilkhu, T. Drew, A. Sheppard, The influence of end of day silicone hydrogel daily disposable contact lens fit on ocular comfort, physiology and lens wettability, Cont Lens Anterior Eye 38 (2015) 339-344.

[36] J. Woods, C. Woods, D. Fonn, Visual performance of a multifocal contact lens versus monovision in established presbyopes, Optom Vis Sci 92 (2015) 175-183.

[37] A. Sivardeen, D. Laughton, J. S. Wolffsohn, Randomized Crossover Trial of Silicone Hydrogel Presbyopic Contact Lenses, Optom Vis Sci 93 (2016) 141-149.

[38] P. Chamberlain, P. B. Morgan, K. J. Moody, C. Maldonado-Codina, Fluctuation in visual acuity during soft toric contact lens wear, Optom Vis Sci 88 (2011) 534-538. 